\title{
A physical model for the popsicle stick cobra
}

\author{
Athanasios Papastathopoulos-Katsaros ${ }^{*}$ and Savvas Sardelis \\ University of Crete, Panepistimioupoli Vouton, Heraklion, Crete 70013, Greece
}

Received: 19 December 2016 / Accepted: 15 June 2017

\begin{abstract}
Popsicle stick bombs are easy-to-build wooden constructions that probably reminisce a few funny moments of our childhood. In this work we look deeper in the travelling wave and we examine which parameters affect the height of the wave and how. We investigate the physics of bent materials and derive their potential energy around which we base our theoretical model. We present the results of the experiments that we performed which referred to the Young's modulus, the geometry of the sticks and their maximum height of the jump after the 'explosion'. We compare the experimental results with the theoretical predictions and we discuss these comparisons. Lastly, we propose potential improvements that might increase the precision of our model.
\end{abstract}

Keywords: elasticity / elastic constants / general physics / deformation and plasticity

\section{Introduction}

A stick bomb (or cobra) is a device constructed out of popsicle sticks woven together in a criss-crossing pattern (see Fig. 1). Each popsicle stick is bent and elastic energy is stored in this deformation. The role of the first and the last stick of the construction is to keep the sticks connected to each other. From now on, we will refer to them as the "stoppers". If one of the stoppers is released, the sticks rapidly straighten out, releasing the energy stored in their deformation. This motion propagates to the other end of the construction very quickly, thus creating an effect that resembles a propagating "explosion".

By removing the stopper, all the potential energy stored at the second stick is released. This energy is then converted to kinetic energy and the stick jumps. When the second stick jumps, the third is able to release its own energy and it goes on. The chain reaction starts and this wave motion propagates.

In this article we have two aims:

- to introduce a model for calculating the maximum height of the sticks in a popsicle stick cobra;

- to examine the parameters on which the height of the wave travelling in a popsicle stick bomb depends.

\section{Methods}

\subsection{Theoretical model}

In order to study the behavior of the popsicle stick cobra, we developed our theoretical model by assuming that the popsicle sticks are rectangular rods and bend because of the

\footnotetext{
* e-mail: ph4452@edu.physics.uoc.gr
}

construction of the bomb. Therefore, we can use the bent rod model (see [1]) for calculating the elastic energy of our sticks,

$$
U_{e}=\frac{E b h^{3} \ell}{24 R_{c}^{2}}
$$

(see Appendix A1), where $E, b, h, \ell$ and $R_{c}$ are respectively the Young's modulus, the width, the thickness, the length and the radius of curvature of the sticks. We assume that the radius of curvature is the same for all the sticks, although this is not in phase with the reality. When the stopper is released, the elastic energy of the sticks is converted to kinetic energy, which is then converted to gravitational potential energy, $U_{g}=m g H$, where $m$ is the mass of the stick, $g$ is the gravitational acceleration and $H$ is the height. Assuming that the motion is purely vertical and that there are no losses due to the air or due to collisions with other sticks of the bomb, every time a stick reaches a maximum height, it has no kinetic energy, only gravitational potential energy. We can consider our bomb as a chain reaction and consider for each individual stick that all its elastic energy is converted to gravitational by solving the equation $U_{b}=U_{e}$ which gives the following expression for the maximum height of each stick,

$$
H=\frac{1}{24} \frac{E b h^{3} \ell}{R_{c}^{2} m g} .
$$

On the other hand, there are some factors which are not included above but might be significant. Previously, we assumed that the motion of the individual sticks is purely vertical and that the sticks do not rotate, but most of the 


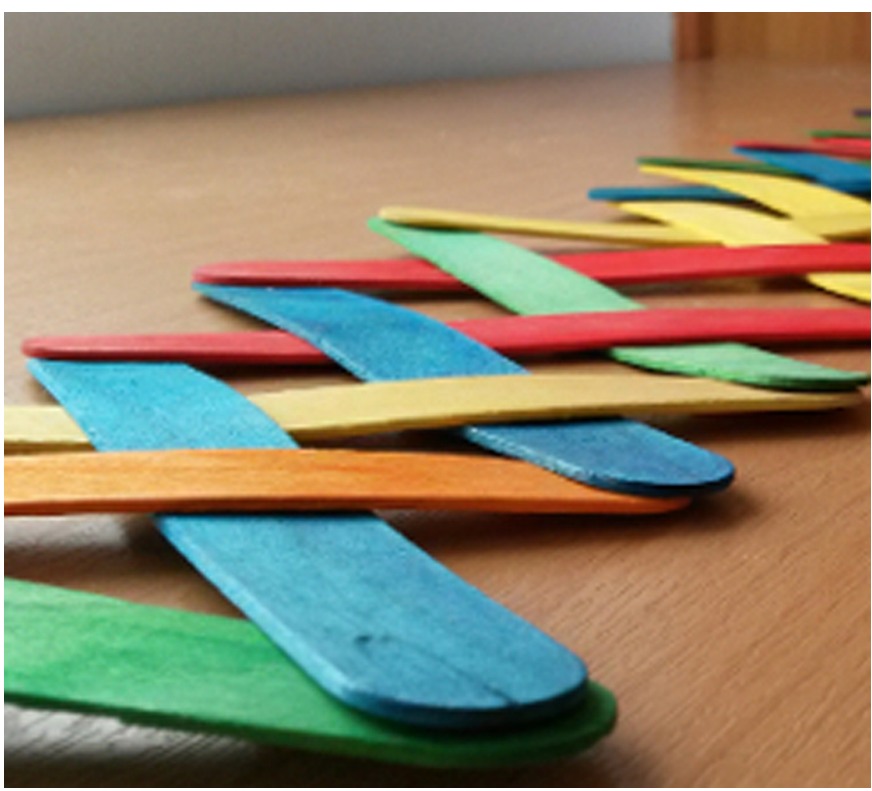

Fig. 1. An example construction of one of our popsicle stick bombs showing the criss-crossing pattern. The photo was taken by us.

time they do. In addition, when the sticks straighten out, an amount of energy is lost when they hit the ground before start jumping, due to the friction between them and the table. Moreover, if they are permanently deformed or if they wobble or if they collide with each other while they are on air, energy will also be lost. We cannot take into account all these unpredictable phenomena that might play a role, so in order to have a more complete model, we include the rotational kinetic energy of the sticks while they reach their maximum height of the jump and the effects of their permanent deformation. The reason why we cannot examine the other effects is partially experimental, as we need a very good experimental setup with a high FPS camera in order to observe the wobbling and analyze the motion of each individual stick.

The sticks do not simply move linearly during their motion, but additionally they rotate. Especially at the maximum height each stick reaches, we assume it is only rotating. As a result, an amount of the initial elastic energy becomes rotational kinetic energy,

$$
U_{r}=\frac{m}{24}\left(b^{2}+\ell^{2}\right) \omega^{2},
$$

(see Appendix A2), where $\omega$ is the angular velocity of the stick. Assuming that the angular velocity is constant because the time interval is small (although the rotational motion takes place around all the axes and around the middle axes it is unstable, see [2]), we can calculate experimentally the angular velocity and subtract the rotational energy from our previous model.

To take into account the losses due to permanent deformation, we should develop a model to calculate this energy of permanent deformation. At first approximation, we assume that we can calculate it by the same formula as
Table 1. Measured properties of large and small sticks.

\begin{tabular}{lll}
\hline Quantity & Values (large) & Values (small) \\
\hline Length & $(12.1 \pm 0.1) \mathrm{cm}$ & $(9.1 \pm 0.1) \mathrm{cm}$ \\
Width & $(0.95 \pm 0.05) \mathrm{cm}$ & $(0.90 \pm 0.05) \mathrm{cm}$ \\
Thickness & $(1.5 \pm 0.1) \mathrm{mm}$ & $(1.8 \pm 0.1) \mathrm{mm}$ \\
Radius of curvature & $(0.3 \pm 0.02) \mathrm{m}$ & $(0.25 \pm 0.02) \mathrm{m}$ \\
Mass & $(1.5 \pm 0.1) \mathrm{g}$ & $(1.3 \pm 0.1) \mathrm{g}$ \\
\hline
\end{tabular}

the one for elastic deformation, equation (1). The difference is that the radius of curvature used to calculate it is the one that the sticks had permanently after only one explosion.

\subsection{Experiments}

We performed experiments to test if our theoretical understanding captures well the physics of the system. Our experiments aimed at answering two specific questions:

Q1) For a given set of $E, b, h, \ell, R_{c}$ and $m$, does equation (2) predict values for the height that are consistent with

Q2) Is the linear dependence of $H$ on $\left(\frac{h^{3} \ell}{R_{c}{ }^{2} m}\right)^{2}$ (see Eq. (2)) verified experimentally?

To answer Q1 we constructed two cobras of length $\sim 3 \mathrm{~m}$. Each cobra was made of a different kind of popsicle sticks. The dimensions of the sticks are shown in Table 1. For one cobra we used sticks (referred to as the "large" sticks), while for the other we used sticks (referred to as the "small" sticks). The errors for the dimensions and the mass were given by the instruments used to measure them (see Tab. 1). The whole popsicle stick explosion was captured by a video camera. In Figure 2 the experimental setup is shown. To measure the heights we built the bombs on a big desk and we calibrated a "ruler" on the blackboard.

The radius of curvature of the bent sticks was measured by taking some pictures of our construction. Thus by using a photo editor we fitted five circles to each different bent stick and the software informed us about their radii in pixels (see Fig. 3). They were converted to meters, as we had calibrated a known length on the photographs. Our results about the radii of curvature of the sticks are presented in Table 1. To measure the Young's modulus of the sticks we assumed that their deformation is elastic. We placed a popsicle stick at the edge of a horizontal surface, see Figures 4 and 5. The stick was fixed at one end with a very heavy load. The other end was free to move vertically. Then, weights of known mass were hung on the free end and as a result, the stick bent and we measured its deflection with the white ruler on the left. The following expression can be used to calculate the Young's modulus,

$$
E=\frac{w \ell^{3}}{3 I \Delta}
$$

(see [3]) where $w=m g$ are the known weights, $I$ is as previously the second moment of area of the cross-sectional area of the stick, $\ell$ is the length and $\Delta$ is the deformation. 


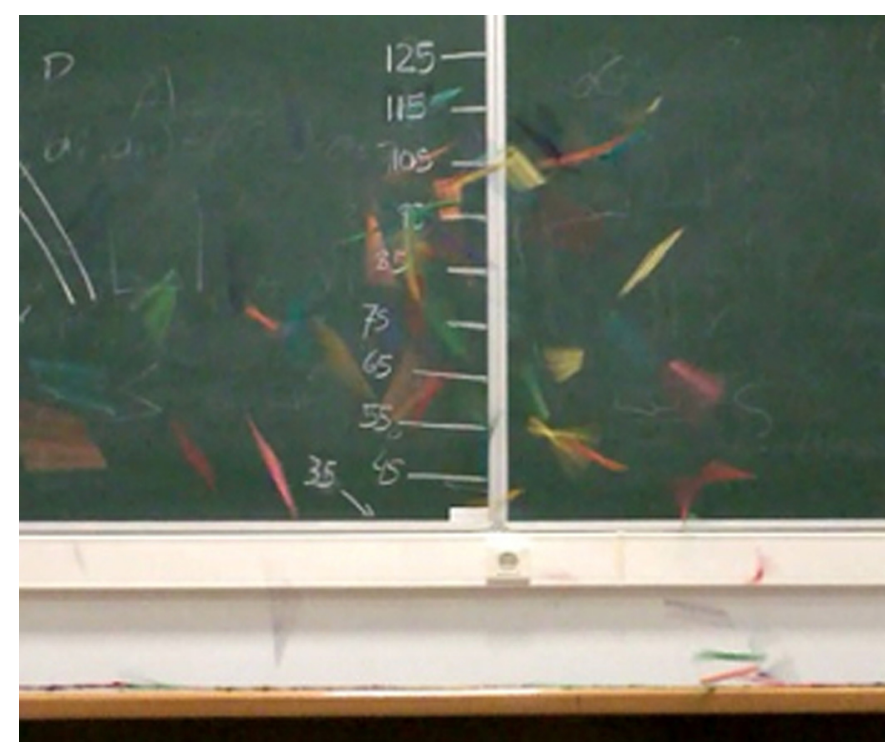

Fig. 2. The first experimental setup for measuring the maximum height of the popsicle stick cobras. The calibrated ruler is shown on the blackboard. This photo is a frame taken from the video that was capturing the popsicle explosion.

The reason we assume that the deformation of the sticks is elastic is that the weights did not stay suspended for long and the deformations were small. The Young's modulus was found to be $(7.1 \pm 0.2) \mathrm{GPa}$ for both types of sticks and corresponds to existing tabulated values (see [4]).

Lastly, we extracted the angular velocity of the ejected sticks by using the short movies of the explosion of a cobra. We measured the time a stick takes to fall to the ground from its maximum height and the number of the rotations it made during its fall. The angular velocity is given by

$$
\omega=\frac{2 \pi N}{\Delta t}
$$

where $N$ is the number of rotations and $\Delta t$ the duration of the fall. Substituting $\Delta t$ and $N$ from our video, we find that the rotational kinetic energy is two orders of magnitude less than the elastic bending energy, and so can be neglected. Lastly, as regards the permanent deformation, we tried to use the method described in Section 2.1, but the radius of curvature of the sticks after only one explosion was almost immeasurable, and as a result the energy of permanent deformation is zero. A potential improvement would be to measure the Young's modulus of the sticks after one explosion, because the radius of curvature might not change, but the sticks could become "softer".

Using our measurements we were able to predict the heights, which were $(0.8 \pm 0.1) \mathrm{m}$ and $(1.7 \pm 0.3) \mathrm{m}$, for the large and the small sticks respectively, see equation (2). The uncertainties result from propagation of measurement errors.

For Q2 we constructed seven different popsicle stick bombs, in order to observe the linear dependence of $H$ on $\left(\frac{b h^{3} \ell}{R_{c}{ }^{2} m}\right)^{2}$ (see Eq. (2)). To achieve that we built them over a desk and we followed the same process. The only exception was the calibration, where we used a known

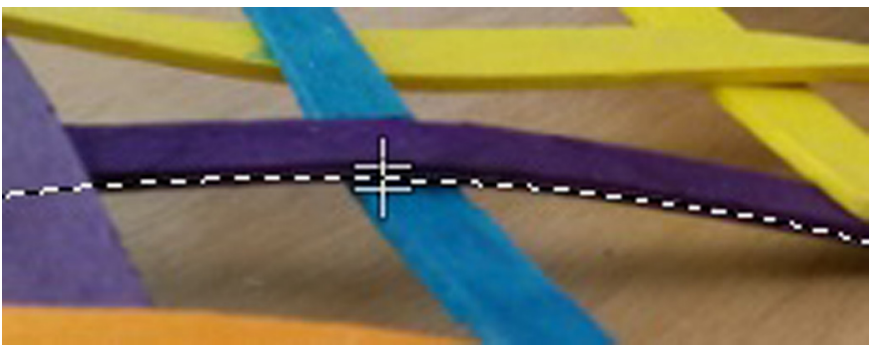

Fig. 3. A demonstration of the method for measuring the radius of curvature. A dashed circle is fitted alongside the bent stick.

length (i.e. a popsicle) in each photograph for the purpose of calibrating the pixels with the length units. By using a constant Young's modulus, the knowledge of its value was unnecessary for these experiments, because it does not alter the linear dependence of the height with respect to $\left(\frac{b h^{3} \ell}{R_{c}{ }^{2} m}\right)^{2}$.

It is possible that some sticks in our experiment had a larger deformation that changed the results. To explore this effect to its limit, we constructed a cobra and let it sit for a week. When removing the stopper, the sticks stayed in place instead of releasing their energy. This effect is caused by plastic deformation, which is irreversible and not instant (see [5]). We conclude that the effect of permanent deformation can be important and must be taken into account if sticks are used repetitively in cobras.

\section{Results}

The maximum heights of the popsicle stick cobras are shown in Table 2. We measured twenty different values of maximum heights, by using different frames of the videos, and calculated their mean average and their standard deviation value for the uncertainties. By contrasting the theoretical heights with the experimental ones for each popsicle cobra (see Tab. 2), we can calculate the relative error given by

$$
d=\frac{\left|H_{t h}-H_{\exp }\right|}{\sqrt{\delta H_{t h}^{2}+\delta H_{\exp }^{2}}},
$$

(see [6]) where the "th" indices refer to the theoretical values and the "exp" to the experimental ones. In our case, this difference was $1.1 \sigma$ for the large sticks and $0.9 \sigma$ for the small ones. Our results are marginally consistent with the theoretical predictions. The fact that the theoretical value is larger than the experimental is reasonable, because we have not taken into account any of the losses that will lower the expected value.

\section{Discussion}

In this work, we made some simplifying assumptions. First, we assumed that the popsicle sticks had a perfect rectangular shape, although in reality they had rounded corners. The expected changes should not be significant, 


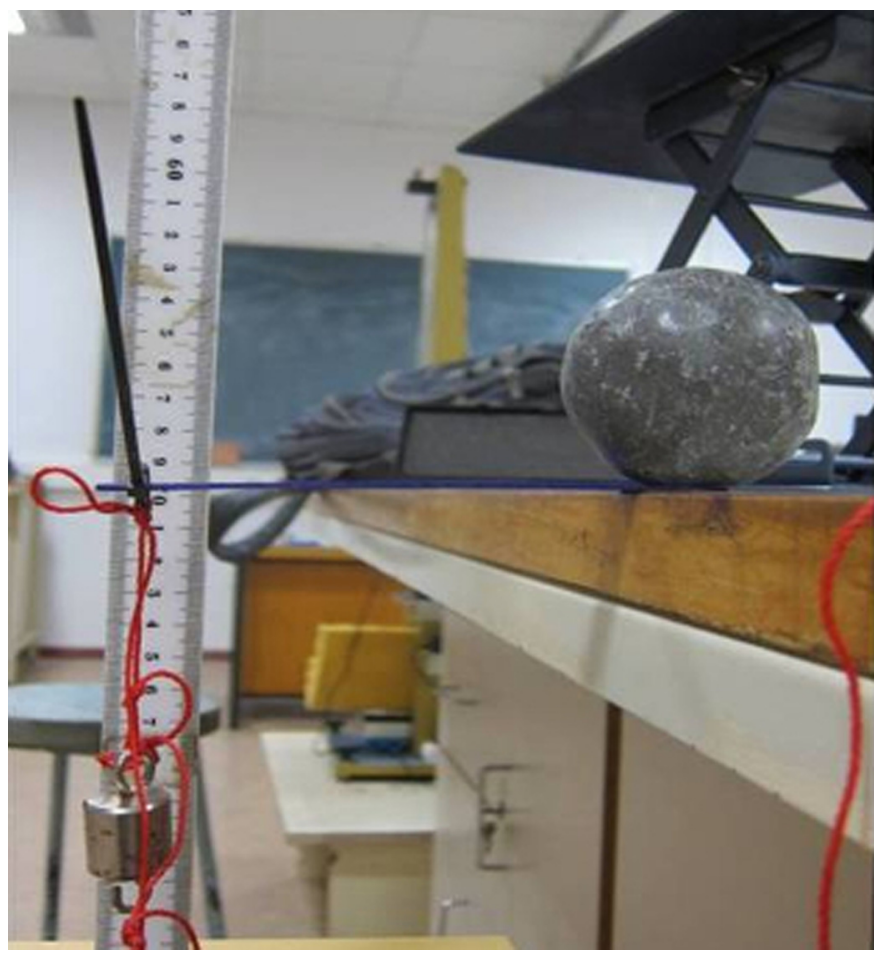

Fig. 4. The experimental setup for measuring the Young's modulus. On the right there is the load that kept the one end of the stick fixed and on the left there are the suspended known weights and a ruler for measuring the deflection of the stick. The uncertainty of the ruler was $1 \mathrm{~mm}$.

since our theory matches the experiments. To consider a more detailed shape of the popsicle stick, we should use a more complete bent plate model, instead of a simple bent rod model, to use the fact that the corners are rounded. We proposed that the main mechanism of the explosion is the conversion of elastic bending energy to kinetic and then to gravitational potential and we verified our theoretical model about the parameters that influence the maximum height a popsicle stick cobra can reach.

During our experiments we found that sticks acquire a small deformation after being part of a cobra. Although we could not measure this deformation, we could not test all the sticks. In addition, we found that sticks that are repeatedly used for building popsicle stick cobras should not be used, as they are significantly deformed permanently.

The last factor that we consider is the base on which the popsicle cobra explodes. In our experiment we used a rigid wooden table. We suggest a different material to be used as the base in future research, to determine any relation between the cobras height and the base on which the bomb explodes.

\section{Dead ends}

In order to answer Q2, we needed a large variety of sticks. Due to lack of resources, we were only able to acquire $\sim 40$ sticks which were only enough to make $50 \mathrm{~cm}$-long cobras.

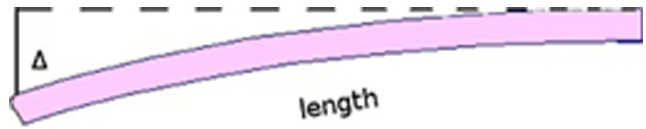

Fig. 5. A sketch of a stick explaining the experimental setup for measuring the Young's modulus in Fig. 4. $\Delta$ is the deflection. The stick's length is subtitled.

Table 2. A comparison between the experimental and the theoretical values of the heights.

\begin{tabular}{lll}
\hline Stick & Exper. height $(\mathrm{m})$ & Theor. height $(\mathrm{m})$ \\
\hline Large & $0.6 \pm 0.1$ & $0.9 \pm 0.2$ \\
Small & $1.3 \pm 0.3$ & $1.8 \pm 0.4$ \\
\hline
\end{tabular}

We discovered that such short cobras are problematic. Consecutive measurements of the maximum height of the cobra did not produce consistent results. Furthermore, the first sticks in the line reached systematically lower heights than those at the end (e.g. $40 \mathrm{~cm}$ versus $2 \mathrm{~m}$ ). We were thus unable to test the linear dependence of $H$ on $\left(\frac{b h^{3} \ell}{R_{c}{ }^{2} m}\right)^{2}$.

\section{Conclusion}

In an effort to elucidate the parameters that characterize the maximum height a popsicle cobra reaches, we developed a theoretical model and performed two experiments. The main parameters found to influence the jump height are the Young's modulus of the sticks, their dimensions, their radius of curvature when they are bent and the gravitational acceleration, see equation (2). We manage to answer Q1 for one specific type of popsicle sticks, but by lack of time and means, we did not manage to test the linear dependency asked in Q2. Future experiments should use long stick bombs for testing this scaling.

An improvement that can be performed in future research is to include to the theoretical model all the parameters mentioned in the text, as well as other factors not discussed in here, such as the reaction force from the table. Lastly, dynamical studies are also very important. They might help understanding the physics behind the popsicle stick bombs, because on videos, we clearly see that the velocity is constant for the whole journey of the cobra, which is not in phase with the big differences in heights.

We acknowledge the whole Greek team of International Physicists Tournament 2016 and all those who volunteered in the experimental procedure. Especially, we thank the team leader G. Panopoulou for her precious advice for solving the problem throughout the sessions and for the writing of the article. We are also grateful to professors K. Tassis and V. Pavlidou for their valuable discussions about the problem. 


\section{Appendices}

\section{A.1 Bent rod}

The elastic energy of a rectangular bent rod is (see [1])

$$
U_{e}=\frac{E I \ell}{2 R_{c}^{2}},
$$

where $E$ is the Young's modulus of the rod, $I$ is the second moment of area of the cross-sectional area, $\ell$ is the length of the rod and $R_{c}$ is the radius of curvature of the bent rod. The second moment of area is given by

$$
I=\iint_{R} \rho^{2} d A
$$

For the cross-sectional area specifically, we have

$$
I=\int_{-b / 2}^{b / 2} \int_{-h / 2}^{h / 2} y^{2} d y d x
$$

which finally leads to

$$
I=\frac{b h^{3}}{12}
$$

where $b$ is the width of the stick and $h$ is its thickness.

Therefore, by combining equations (A1) and (A2) we arrive at equation (1).

\section{A.2 Rotational kinetic energy}

The rotational kinetic energy of an object is

$$
U_{r}=\frac{1}{2} I \omega^{2} .
$$

The moment of inertia around the center of mass for a very thin rectangular plate $(h \approx 0)$ is given by (see [7])

$$
I=\frac{1}{12} m\left(b^{2}+\ell^{2}\right),
$$

and by combining equations (A3) and (A4) we arrive at equation (3).

\section{References}

1. L.D. Landau, E.M. Lifshitz, Theory of Elasticity (Pergamon Press, Oxford, UK, 1959), p. 75

2. J.E. Marsden, T.S. Ratiu, Introduction to Mechanics and Symmetry (Springer, New York, USA, 1998), p. 490

3. J.M. Gere, Mechanics of Materials (Nelson Thornes, Cheltenham, UK, 2004), p. 620

4. In: Wood Handbook: Wood as an Engineering Material, edited by Z. Cai, R.J. Ross (Forest Products Laboratory, Madison, USA, 2010), Vol. 12, p. 12-2

5. W.D. Callister, Fundamentals of Materials Science and Engineering (John Wiley and Sons Inc., Hoboken, NJ, USA, 2004), p. 143

6. P.R. Bevington, D.K. Robinson, Data Reduction and Error Analysis for the Physical Sciences (McGraw-Hill, New York, USA, 1994)

7. R.A. Serway, J.W. Jewett, Physics for Scientists and Engineers with Modern Physics (Thomson, Stamford, CT, USA, 2008), p. 278

Cite this article as: Athanasios Papastathopoulos-Katsaros, Savvas Sardelis, A physical model for the popsicle stick cobra, Emergent Scientist 1, 3 (2017) 